\title{
Preeclampsia: su asociación con infecciones periodontales y urinarias según trimestre del embarazo
}

\author{
Preeclampsia: its association with periodontal and urinary \\ infections according to trimester of pregnancy
}

\section{Jorge Alejandro Lopera Rodríguez ${ }^{1 \otimes \underline{\text { CvLAC}}}$, Emérita Rocha Olivera²}

1. Médico y Cirujano, Especialista en Epidemiología. Grupo de investigación GINECOCES, Universidad CES. Corporación IPS Comfamiliar Camacol Coodán. Medellín, Colombia.

2. Enfermera, Especialista en Epidemiología. Grupo de investigación GINECOCES, Universidad CES. Medellín, Colombia.

Este trabajo cumple con las siguientes características editoriales

Fecha correspondencia: Recibido: diciembre 4 de 2015. Revisado: marzo 7 de 2016. Aceptado: marzo 9 de 2016.

Forma de citar:

Lopera-Rodríguez JA, RochaOlivera E. Preeclampsia: su asociación con infecciones periodontales y urinarias según trimestre del embarazo. Rev CES Med 2016. 30(1): 14-25.

Open access

(C) Derecho de autor

Licencia creative commons

Etica de publicaciones

Revisión por pares

Gestión por Open Journal

System

ISSN 0129-8705

ISSN-e 2215-9177

Comparte

\section{Resumen}

Introducción: la preeclampsia es un problema que afecta la salud materna y puede causar la muerte de la gestante. Su etiología aún es desconocida. Uno de los factores asociados con la preeclampsia es el de las infecciones en el embarazo, pero no se ha caracterizado su papel en cada trimestre de la gestación. El objetivo del presente estudio fue reconocer qué asociación existe entre las infecciones según su presentación en cada trimestre del embarazo y la preeclampsia. Métodos: estudio de 85 casos y 85 controles de registros de historias clínicas, apareados por antecedentes ginecobstétricos, edad e índice de masa corporal al principio del embarazo. Se realizaron análisis univariado, bivariado y multivariado. Resultados: no se encontró asociación con rinofaringitis, infección urinaria diagnosticada clínicamente o por urocultivo, ni para urocultivo positivo. Un comportamiento similar se observó en el análisis de cada trimestre. En el análisis multivariado, la presencia de caries en el primer trimestre, ajustada por variables relacionadas con infección urinaria mostró una asociación de riesgo con un OR de 2,04. Conclusiones: la caries en el primer trimestre del embarazo parece tomar relevancia como factor de riesgo para preeclampsia cuando ocurre concomitantemente con infecciones urinarias y otros hallazgos relacionados con infección dental.

Palabras clave: Preeclampsia, Infecciones periodontales, Infecciones urinarias.

\footnotetext{
Abstract

Introduction: preeclampsia is a problem that affects maternal health and can cause death in pregnant women. Therefore, preeclampsia is considered a public health problem. Despite, the etiology of preeclampsia is unknown yet. A factor associated with preeclampsia is infections in pregnancy, but the role of this factor has not been characterized by pregnancy trimester. The main goal of this work is to determine if there is an association between infections in each trimester of pregnancy and preeclampsia.
} 
En 2013, fue definida por el Colegio Americano de Obstetricia y Ginecología, como la presencia de presión arterial mayor a 140/90 $\mathrm{mm} / \mathrm{Hg}$ en dos momentos con cuatro horas de diferencia después de la semana 20 de gestación, en una mujer con una presión sanguínea previamente normal y presencia de proteinuria determinada en orina de 24 horas de 300 mg, relación proteínas/ creatinina mayor de 0,3 $\mathrm{mg} / \mathrm{dl}$ o en lectura en tirilla de una cruz en orina si otros métodos no están disponibles.
Methods: this study was performed using cases and controls methodology. 85 cases and 85 controls were obtained from clinical registers. These were appeared by gynecobstetric history, age and body mass index. A univariate analysis was performed to characterize the patients in the study. A bivariated analysis was done to know the individual association between infections and preeclampsia. Finally, a multivariated analysis was applied. Results: it was not founded association with rinofaringitis, tract urinary infection diagnosed through clinical and urine culture and positive urine culture. A similar behavior was observed in the analysis by trimester. In the multivariated analysis adjusted by tract urinary infection variables and dental findings, the presence of caries in the first trimester showed a risk association with OR 2,04. Conclusions: caries in the first trimester takes relevance when it occurs at the same time that tract urinary infection and other findings associated to dental infections.

Keywords: Preeclampsia, Periodontal infections, Urinary tract infections.

\section{Introducción}

Cerca de medio millón de mujeres mueren cada año por causas relacionadas con el embarazo y el $99 \%$ de estas ocurren en países con ingresos bajos o medios (1). La hemorragia obstétrica y los desórdenes hipertensivos del embarazo fueron las mayores causas conocidas de muerte materna alrededor del mundo entre 2003 a 2009 (2); en 2013 los desórdenes hipertensivos en el embarazo fueron la segunda causa de muerte materna en Latinoamérica andina con un promedio de 174 casos, teniendo como primera causa la muerte materna tardía con un promedio de 260 casos (3).

En Colombia, entre 2012 a 2013 del total de muertes maternas ocurridas, el 18,2 \% fueron por desórdenes hipertensivos del embarazo, siendo la segunda causa conocida de muerte materna en el país (4).

Entre los trastornos hipertensivos asociados al embarazo se encuentra la preeclampsia, que puede presentarse en cualquier momento de la gestación al alcanzarse la semana 20 (ㄷ) y que puede evolucionar a eclampsia, entidad clínica que pone en riesgo la vida de la paciente.

En 2013, la preeclampsia fue definida por el Colegio Americano de Obstetricia y Ginecología, por sus siglas en inglés (ACOG) como la presencia de presión arterial mayor a $140 / 90 \mathrm{~mm} / \mathrm{Hg}$ en dos momentos con cuatro horas de diferencia después de la semana 20 de gestación, en una mujer con una presión sanguínea previamente normal y presencia de proteinuria determinada en orina de 24 horas de $300 \mathrm{mg}$. relación proteínas/creatinina mayor de $0,3 \mathrm{mg} / \mathrm{dl}$ o en lectura en tirilla de una cruz en orina si otros métodos no están disponibles (므.

A falta de proteinuria también se tienen en cuenta para el diagnóstico factores como trombocitopenia por debajo de 100000 plaquetas por microlitro, desarrollo de disfunción renal reconocida como creatinina mayor de 1,1 mg/dl, disfunción hepática definida como aumento de transaminasas al doble de lo normal, edema pulmonar y aparición de síntomas cerebrales o visuales (므).

La búsqueda por identificar las causas de la preeclampsia se ha realizado en todas partes del mundo encontrándose múltiples asociaciones de factores de riesgo y su

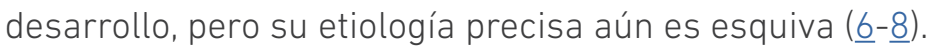


Uno de estos factores estudiados es el de las infecciones durante el embarazo, siendo la infección urinaria y la enfermedad periodontal las principales entidades infecciosas que podrían estar implicadas.
Uno de estos factores estudiados es el de las infecciones durante el embarazo (q11), siendo la infección urinaria y la enfermedad periodontal las principales entidades infecciosas que podrían estar implicadas (10). La primera se define como la presencia de microorganismos patógenos en el tracto urinario, ya sea con sobrecrecimiento en orina, generación de síntomas o ambas (12); mientras que la segunda se define como la invasión de bacterias en el periodonto con formación de una biopelícula lo que causa una respuesta inflamatoria crónica (13) que puede llevar a la pérdida del diente.

A pesar de que estas afecciones pueden ser muy comunes durante el embarazo, la evaluación de su asociación con el síndrome preeclámptico en nuestra región aún es escasa; igualmente, los estudios que evalúen la asociación según el momento de presentación durante el embarazo.

Lo anterior lleva a la necesidad de ampliar los conocimientos al respecto, con el fin de reconocer cómo se modifica el riesgo de preeclampsia dependiendo de si la infección se presenta en el primer, segundo o tercer trimestre del embarazo.

\section{Metodología}

Estudio de casos y controles. La muestra se calculó tomando el odds ratio (OR) de efectos aleatorios para enfermedad periodontal de 2,37 (10), con un nivel de confianza de $90 \%$, un poder del $80 \%$, un porcentaje de exposición de los controles del 20 \%, una relación caso: control de 1: 1. Se utilizó Epidat versión 3.1 para realizar el cálculo. El cálculo dio una muestra de 85 casos y 85 controles.

El estudio se desarrolló con registros de pacientes del Valle de Aburrá y municipios aledaños quienes fueron atendidas en sus controles prenatales en la institución prestadora de salud Corporación IPS Comfamiliar Camacol - Coodán (CICCC) entre los años 2010 a 2015, que atiende a pacientes afiliados a las empresas promotoras de salud (EPS) pertenecientes a Cafesalud, Cruz Blanca y Saludcoop.

La obtención de los casos fue realizada mediante búsqueda electrónica en los registros individuales de prestación de servicios de salud de la institución. La búsqueda se realizó utilizando los códigos del Manual Diagnóstico de Enfermedades CIE-10 (14) desde el 010X al 016X, que están relacionados con edema, proteinuria e hipertensión en el embarazo, el parto y el puerperio.

Una vez seleccionado el grupo candidato de pacientes se analizó si cumplían con los criterios para diagnóstico de preeclampsia: presión arterial alta tomada en dos momentos diferentes y proteinuria con más de 300 mg de proteínas en una muestra de orina de 24 horas. Posteriormente se analizó si el grupo restante cumplía con los criterios de caso para inclusión en el estudio: que hubieran sido adscritas para su atención a la CICCC desde tres meses antes del embarazo, durante su embarazo y hasta tres meses después del parto; que en la historia se pudieran corroborar los datos clínicos y de laboratorio que llevaron al diagnóstico de preeclampsia; que hubieran realizado al menos un control prenatal por trimestre durante su embarazo; que hubieran realizado al menos una visita por odontología durante el embarazo; y que hubieran realizado al menos un urocultivo durante el embarazo.

Los criterios de inclusión de los controles fueron iguales a los de los casos excepto por la presentación de preeclampsia. Estos fueron obtenidos de la base de datos de 
Para el diagnóstico de las infecciones del tracto urinario (ITU) se tuvo en cuenta la sintomatología clínica y el aislamiento en urocultivo de más de 100000 unidades formadoras de colonias de bacterias.
Para la evaluación del compromiso oral se tuvieron en cuenta los siguientes hallazgos y diagnósticos obtenidos por el odontólogo en el examen prenatal: caries, gingivitis, pulpitis, periodontitis, placa bacteriana blanda y placa bacteriana dura o calcificada (cálculos). atención prenatal. Ambos grupos fueron pareados usando las variables: edad \pm 3 años, antecedentes ginecobstétricos e índice de masa corporal al principio del embarazo según la siguiente clasificación: bajo peso menor o igual de $19,9 \mathrm{~kg} / \mathrm{m}^{2}$, peso normal de 20 a $24,9 \mathrm{~kg} / \mathrm{m}^{2}$, sobrepeso de 25 a $29,9 \mathrm{~kg} / \mathrm{m}^{2}$, obesidad grado / de 30 a $34,9 \mathrm{~kg} / \mathrm{m}^{2}$ y obesidad grado $/ /$ de $35 \mathrm{~kg} / \mathrm{m}^{2}$ en adelante.

Por dificultades en obtener información acerca del estrato socioeconómico se incluyó en su lugar la variable nivel salarial, que refleja indirectamente la situación socioeconómica del núcleo familiar. El nivel salarial se definió en base a lo contenido en el acuerdo 260 de 2004 del Consejo Nacional de Seguridad Social en Salud de Colombia así: nivel salarial 1, ingresos menores a dos salarios mínimos legales mensuales vigentes (SMLMV); 2: ingresos entre dos y cinco SMLMV y nivel salarial 3: ingresos mayores de cinco SMLMV. Dicha información fue obtenida de la propia historia clínica.

Para el diagnóstico de las infecciones del tracto urinario (ITU) se tuvo en cuenta la sintomatología clínica y el aislamiento en urocultivo de más de 100000 unidades formadoras de colonias de bacterias. Dado que los síntomas no siempre se acompañaron de un urocultivo positivo o de la realización de alguno, para el análisis se plantearon dos grupos: uno constituido por todas las pacientes que presentaron diagnósticos clínicos o que presentaron hallazgos positivos de laboratorio y el segundo, más restringido, constituido por las pacientes que presentaron urocultivo positivo y que no necesariamente tenían síntomas. El primer grupo se denominó infección del tracto urinario clínica o por urocultivo y el segundo, urocultivo positivo.

Para la definición de rinofaringitis se tuvo en cuenta el diagnóstico que hubiese sido registrado por el médico y que presentara al menos congestión nasal, fiebre y malestar general.

Para la evaluación del compromiso oral se tuvieron en cuenta los siguientes hallazgos y diagnósticos obtenidos por el odontólogo en el examen prenatal: caries, gingivitis, pulpitis, periodontitis, placa bacteriana blanda y placa bacteriana dura o calcificada (cálculos).

Se realizó análisis estadístico univariado. A las variables cuantitativas se les calculó medidas de tendencia central, dispersión y posición, mientras que a las variables cualitativas, frecuencias y proporciones. Posteriormente fue realizado el análisis bivariado para determinar la asociación de las variables independientes propuestas (rinofaringitis, infección del tracto urinario y hallazgos odontológicos) con la preeclampsia. La significancia estadística fue determinada a través de la prueba de Chi cuadrado y según el número de pacientes en cada análisis se usó el test estadístico de Pearson o el de Fisher.

Finalmente, se realizó un análisis multivariado a través de regresión logística binaria con aquellas variables que mostraran valores de $\mathrm{p}<0,2$. Todos los análisis estadísticos fueron realizados con el software SPSS (R) versión 22.

Los errores del observador y de lo observado buscaron evitarse realizando una revisión exhaustiva de todos los registros clínicos de las atenciones médicas prestadas a cada paciente en consulta externa, urgencias y hospitalización durante su control prenatal, parto, tres meses antes de su embarazo y tres meses después del parto. 
En un análisis inicial de los procesos infecciosos en el embarazo y en el cual no se discriminó el trimestre en que se presentaron, se obtuvo para urocultivo positivo un OR de 0,65 (IC95 \%=0,31-1,38), para ITU diagnosticada por clínica o urocultivo un OR 0,63 (0,33-1,23) y para rinofaringitis de 1,49 (0,77-2,92). Posteriormente se procedió a realizar el análisis por trimestre del embarazo.
El sesgo de información trató de limitarse a través de la participación del seguimiento clínico de la mayor parte de los controles por parte de los investigadores. En el seguimiento clínico de cada paciente casi siempre participaron más de cuatro clases de profesionales (odontólogos, enfermeros, médicos de urgencias, nutricionistas, médicos de control prenatal y médicos ginecobstetras). Todos ellos utilizaron la misma plataforma de software de gestión de datos clínicos (HeOn) de la cual se extrajeron los datos.

Se obtuvieron 770 pacientes con diagnósticos relacionados con síndrome hipertensivo asociado al embarazo, 164 tuvieron criterios clínicos para preeclampsia y 85 cumplieron los criterios de inclusión. El apareamiento de casos y controles se logró en un $100 \%$ por edad, en un 98,8 \% por antecedentes gineco-obstétricos y en un 90,6 \% según índice de masa corporal al principio del embarazo.

El proyecto se acogió bajo la ley 1581 de protección de datos de Colombia en 2012; además tuvo en cuenta los principios éticos: autonomía, no maleficencia, justicia y beneficencia $(\underline{15}, \underline{16})$. La identidad de cada paciente fue protegida y sus datos personales se mantuvieron bajo estricta confidencialidad.

El financiamiento de este proyecto estuvo a cargo de la CICCC. Los autores declaran que no tuvieron situaciones particulares que causaran conflicto de intereses con el desarrollo de la presente investigación.

\section{Resultados}

La muestra estuvo constituida principalmente por pacientes de etnia mestiza con estudios superiores y en el nivel salarial 1 (cuadro 1).

El 28,8 \% de las pacientes del estudio presentaron infección del tracto urinario diagnosticada por clínica o urocultivo, frente a $21,2 \%$ que sólo presentaron urocultivo positivo. Durante el primer trimestre no se les realizó urocultivo al $17,6 \%$ de la población, en el segundo trimestre al 45,2 \% y en el tercer trimestre al 55,3\%. La rinofaringitis se presentó en el $28,8 \%$ de las pacientes.

En el análisis bivariado, inicialmente se cruzaron las variables sociodemográficas con la preeclampsia (cuadro 2). Se omitieron las variables de edad, índice de masa corporal al principio del embarazo y antecedentes gineco-obstétricos, ya que estaban controladas mediante apareamiento.

En un análisis inicial de los procesos infecciosos en el embarazo y en el cual no se discriminó el trimestre en que se presentaron, se obtuvo para urocultivo positivo un OR de 0,65 (IC95 \%= 0,31-1,38), para ITU diagnosticada por clínica o urocultivo un OR $0,63(0,33-1,23)$ y para rinofaringitis de $1,49(0,77-2,92)$. Posteriormente se procedió a realizar el análisis por trimestre del embarazo (cuadro 3 ).

Se realizó análisis bivariado con los hallazgos y diagnósticos encontrados en el examen odontológico en cada trimestre del embarazo (cuadro 4).

Finalmente, se incluyeron en el análisis multivariado aquellas variables que mostraron significancia estadística con $p<0,2$ (cuadro 5). 
Cuadro 1. Características sociodemográficas.

\begin{tabular}{|c|c|c|c|c|c|}
\hline Característica & Casos & DE o \% & Controles & DE o \% & $\boldsymbol{p}$ \\
\hline Media de la edad & 28,67 & 6,03 & 28,49 & 5,74 & 0,00 \\
\hline Media de IMC al principio del embarazo & 26,05 & 4,39 & 24,32 & 3,76 & 0,00 \\
\hline Nivel de escolaridad & & & & & 0,37 \\
\hline Sin escolaridad & 1 & 1,2 & - & - & \\
\hline Primaria & 5 & 5,9 & 2 & 2,4 & \\
\hline Secundaria & 33 & 38,8 & 31 & 36,5 & \\
\hline Estudios superiores & 46 & 54,1 & 52 & 61,2 & \\
\hline Nivel Salarial & & & & & 0,43 \\
\hline Nivel 1 & 66 & 77,6 & 62 & 72,9 & \\
\hline Nivel 2 & 14 & 16,5 & 20 & 23,5 & \\
\hline Nivel 3 & 5 & 5,9 & 3 & 3,5 & \\
\hline Etnia & & & & & 0,01 \\
\hline Mestiza & 67 & 78,8 & 75 & 88,2 & \\
\hline Afrodescendiente & 5 & 5,9 & 2 & 2,4 & \\
\hline Blanca & 5 & 5,9 & - & - & \\
\hline Sin dato & 8 & 9,4 & 8 & 9,4 & \\
\hline Antecedente gestacional & & & & & 0,85 \\
\hline Primigestante & 50 & 58,8 & 50 & 58,8 & \\
\hline Secundigestante & 22 & 25,9 & 25 & 29,4 & \\
\hline Trigestante & 8 & 9,4 & 5 & 5,9 & \\
\hline Multigestante & 5 & 5,9 & 5 & 5,9 & \\
\hline Antecedente personal de hipertensión & & & & & 0,00 \\
\hline Sin antecedente & 72 & 84,7 & 84 & 98,8 & \\
\hline Con antecedente & 13 & 15,3 & 1 & 1,2 & \\
\hline
\end{tabular}

Para las variables cuantitativas edad e IMC al principio del embarazo se presenta la media y la desviación estándar (DE). Para estas dos variables se realizó prueba estadística de t de student. Para las demás variables se presenta el porcentaje que ocupa el valor de la variable en cada grupo y se realizó prueba estadística de Chi cuadrado.

-: Sin casos en la variable.

Cuadro 2. Asociación de preeclampsia según factores sociodemográficos

\begin{tabular}{llccccc}
\hline \multicolumn{2}{l}{ Variables sociodemográficas } & Casos $\%$ & Controles\% & $\boldsymbol{p}$ & OR & IC 95 \% \\
\hline \multirow{2}{*}{ Nivel de } & Sin escolaridad & 100 & - & $1^{\text {a }}$ & 2,01 & $1,73-2,34$ \\
escolaridad & Primaria & 71,4 & 28,6 & $0,44^{\text {a }}$ & 2,59 & $0,49-13,76$ \\
& Secundaria & 51,6 & 48,4 & 0,75 & 1,11 & $0,59-2,06$ \\
& Estudios & 46,9 & 53,1 & 0,35 & 0,75 & $0,41-1,38$ \\
\multirow{2}{*}{ Nivel salarial } & superiores & 51,6 & 48,4 & 0,48 & 1,29 & $0,64-2,59$ \\
& 1 & 41,2 & 58,8 & 0,25 & 0,64 & $0,30-1,37$ \\
\multirow{5}{*}{ Etnia } & 2 & 62,5 & 37,5 & $0,72^{\text {a }}$ & 1,71 & $0,40-7,39$ \\
& Mestiza & 47,2 & 52,8 & 0,10 & 0,50 & $0,21-1,15$ \\
& Afrodescend & 71,4 & 28,6 & $0,44^{\mathrm{a}}$ & 2,59 & $0,49-13,76$ \\
\hline
\end{tabular}

a. Test de Fisher 
Enero - Junio 2016 - Pág 20

Cuadro 3. Asociación de preeclampsia por infecciones en cada trimestre del embarazo del grupo de estudio

\begin{tabular}{lcccccc}
\hline $\begin{array}{l}\text { Diagnóstico relacionado con } \\
\text { infección }\end{array}$ & Trimestre & Casos $\%$ & Controles \% & p & OR & IC 95 \% \\
\hline & Primero & 36,4 & 63,6 & 0,35 & 0,55 & $0,16-1,95$ \\
Rinofaringa & Segundo & 62,5 & 37,5 & 0,19 & 1,81 & $075-4,40$ \\
& Tercero & 57,9 & 42,1 & 0,47 & 1,43 & $0,55-3,76$ \\
& Primero & 48,5 & 51,5 & 0,85 & 0,93 & $0,43-1,98$ \\
ITUb por clínica o por urocultivo & Segundo & 33,3 & 66,7 & 0,14 & 0,46 & $0,17-1,30$ \\
& Tercero & 33,3 & 66,7 & 0,18 & 0,47 & $0,15-1,44$ \\
& Primero & 41,7 & 58,3 & 0,38 & 0,68 & $0,28-1,62$ \\
Urocultivo positivo & Segundo & 30,0 & 70,0 & 0,19 & 0,41 & $0,10-1,63$ \\
& Tercero & 45,5 & 54,5 & 0,76 & 0,82 & $0,24-2,81$ \\
\hline
\end{tabular}

${ }^{a}$ Rinofaring: rinofaringitis. ${ }^{b} \mathrm{ITU}$ : infección del tracto urinario

Cuadro 4. Asociación de preeclampsia con hallazgos y diagnósticos encontrados en el examen odontológico en cada trimestre

\begin{tabular}{|c|c|c|c|c|c|c|}
\hline Hallazgo & Trimestre & Casos \% & Controles \% & $p$ & $O R$ & IC $95 \%$ \\
\hline \multirow{3}{*}{ Caries } & 1 & 56,7 & 43,3 & 0,20 & 1,51 & $0,80-2,85$ \\
\hline & 2 & 54,8 & 45,2 & 0,48 & 1,29 & $0,64-2,59$ \\
\hline & 3 & 37,5 & 65,5 & 0,29 & 0,57 & $0,20-1,65$ \\
\hline \multirow{3}{*}{ Gingivitis } & 1 & 51,2 & 48,8 & 0,76 & 1,10 & $0,60-2,01$ \\
\hline & 2 & 59,3 & 40,7 & 0,10 & 1,73 & $0,90-3,33$ \\
\hline & 3 & 52,9 & 47,1 & 0,80 & 1,14 & $0,42-3,11$ \\
\hline \multirow{3}{*}{$\begin{array}{l}\text { Placa bacteriana } \\
\text { blanda }\end{array}$} & 1 & 47,9 & 52,1 & 0,54 & 0,83 & $0,45-1,51$ \\
\hline & 2 & 56,0 & 44,0 & 0,31 & 1,41 & $0,73-2,73$ \\
\hline & 3 & 52,9 & 47,1 & 0,80 & 1,14 & $0,42-3,11$ \\
\hline \multirow{3}{*}{ Placa bacteriana dura } & 1 & 47,2 & 52,8 & 0,62 & 0,85 & $0,443-1,62$ \\
\hline & 2 & 59,3 & 40,7 & 0,29 & 1,56 & $0,68-3,6$ \\
\hline & 3 & 40,0 & 60,0 & 0,51 & 0,65 & $0,18-2,39$ \\
\hline \multirow{3}{*}{ Pulpitis } & 1 & 50,0 & 50,0 & $1^{\mathrm{a}}$ & 1 & $0,14-7,27$ \\
\hline & 2 & 80,0 & 20,0 & $0,37^{\mathrm{a}}$ & 4,15 & $0,45-37,91$ \\
\hline & 3 & 50,0 & 50,0 & $1^{\mathrm{a}}$ & 1 & $0,06-16,25$ \\
\hline \multirow{3}{*}{ Periodontitis } & 1 & 100 & - & $0,25^{a}$ & 2,04 & $1,75-2,38$ \\
\hline & 2 & 100 & - & $0,50^{\mathrm{a}}$ & 2,02 & $1,74-2,36$ \\
\hline & 3 & 100 & - & $0,25^{\mathrm{a}}$ & 2,04 & $1,75-2,38$ \\
\hline
\end{tabular}

-: cero número de casos en la variable. a: Aplicado test estadístico de Fisher 
Cuadro 5. Análisis multivariado para variables relacionadas con ITU y enfermedad dental

\begin{tabular}{|c|c|c|c|c|}
\hline \multirow{2}{*}{$\begin{array}{l}\text { Variables relacionadas con ITU y enfermedad } \\
\text { dental }\end{array}$} & \multirow{2}{*}{ OR Crudo } & \multirow{2}{*}{ OR Ajustado } & \multicolumn{2}{|c|}{ IC $95 \%$} \\
\hline & & & Mín. & Máx. \\
\hline ITU por clínica o urocultivo trimestre 2 & 0,46 & 0,37 & 0,07 & 2,03 \\
\hline ITU por clínica o urocultivo trimestre 3 & 0,42 & 0,38 & 0,10 & 1,53 \\
\hline Urocultivo positivo trimestre 2 & 0,41 & 0,80 & 0,09 & 7,31 \\
\hline Urocultivo no realizado trimestre 2 & 0,65 & 0,85 & 0,39 & 1,83 \\
\hline Urocultivo no realizado trimestre 3 & 0,42 & 0,33 & 0,13 & 0,85 \\
\hline Urocultivo sin crecimiento trimestre 3 & 2,97 & 1,34 & 0,45 & 3,99 \\
\hline Urocultivo crecimiento polimicrobiano trimestre 2 & 2,27 & 1,97 & 0,71 & 5,43 \\
\hline Caries trimestre 1 & 1,51 & $2,05(p=0,048)$ & 1,01 & 4,15 \\
\hline $\begin{array}{l}\text { Gingivitis trimestre } 2 \\
\text { Placa bacteriana calcificada trimestre } 2\end{array}$ & $\begin{array}{l}1,73 \\
2,40\end{array}$ & $\begin{array}{l}1,80 \\
1,76\end{array}$ & $\begin{array}{l}0,84 \\
0,45\end{array}$ & $\begin{array}{l}3,89 \\
6,96\end{array}$ \\
\hline
\end{tabular}

ITU: infección del tracto urinario

Muchos estudios continúan mostrando a la enfermedad periodontal como un factor asociado a preeclampsia.

\section{Discusión}

Se pudo observar que la preeclampsia parece no guardar asociación con la rinofaringitis, la infección del tracto urinario diagnosticada por clínica o urocultivo ni con urocultivo positivo, diferente a lo encontrado por Conde-Agudelo et al., en su metaanálisis (10) quienes reportan asociación de infección del tracto urinario y preeclampsia $(\mathrm{OR}=1,57$; IC $95 \%=1,4-1,7)$. Encontramos una asociación significativa, en aquellas pacientes a quienes no se les realizó urocultivo en el tercer trimestre. Sin embargo, debe considerarse esta asociación como espuria por su no coherencia con la realidad clínica.

En el estudio de Minassian et al., en el que fueron incluidas 1533 casos y 14236 controles de mujeres primigestantes, obtenidas de la base de datos del General Practice Research del Reino Unido, tras ajustar por edad materna, hipertensión pregestacional, diabetes, enfermedad renal y gestación multifetal, se observa que las mujeres con infección urinaria tienen mayor riesgo de sufrir preeclampsia que las que no la presentaron (OR ajustado 1,22 IC95 \%= 1,03-1,45); igualmente, aquellas a las que les fue prescrito un tratamiento antibiótico tuvieron 1,28 veces más riesgo de sufrir preeclampsia que aquellas a las que no se les prescribió; pero no encontraron asociación con infección del tracto respiratorio (17). Estos resultados son similares con los de nuestro estudio, mientras que el OR ajustado para infección urinaria, a pesar de ser significativo, es muy bajo.

Un estudio realizado por Shamsi et al., con mujeres de Pakistán tampoco encuentra asociación con infección del tracto urinario (18), lo que lleva a considerar que dicha asociación podría modificarse según los factores sociodemográficos y genéticos presentes en la población.

Muchos estudios continúan mostrando a la enfermedad periodontal como un factor asociado a preeclampsia, como en el metaanálisis realizado por Huang et al. (11), que reporta un riesgo de 3,7 veces de sufrir preeclampsia frente a las que no presentaron enfermedad periodontal; mientras que la enfermedad periodontal diagnosticada a las 48 horas antes del parto y cinco días después del parto, mostró un OR de 2,68 (IC95 \%= 1,39-5,18) en el primer caso y un OR de 2,22 (IC95 \%= 1,16-4,27) para el segundo (11). 
Uno de los mecanismos fisiopatológicos propuestos es el de la colonización de la placenta por bacterias orales transportadas por vía hematógena causando alteraciones en el desarrollo normal placentario y generando aterosis. Otro mecanismo es el de cambios inmunológicos sistémicos ocasionados por la enfermedad dental que llevan a complicaciones durante el embarazo.
Ha et al., siguieron a 283 pacientes que nunca habían fumado, observándose que a 13 de estas pacientes se les diagnosticó preeclampsia y tuvieron un OR ajustado para periodontitis de 5,56 (IC95 \% $=1,49-20,71)$ (9).

Puede entonces considerarse que la caries en el primer trimestre, junto con la presentación de otros hallazgos dentales y de infecciones urinarias puede estar asociada con preeclampsia. Poca evidencia hay en la literatura al respecto: un estudio realizado por Khader et al. con 115 mujeres con preeclampsia y 230 controles también encuentra dicha asociación, OR 1,15 (IC $95 \%=1,05-1,25$ ) en el análisis bivariado y OR 1,14 (IC $95 \%=1,03-1,27)$ en el análisis multivariado, en el que se incluyeron factores asociados como la edad, nuliparidad y el índice de masa corporal al principio del embarazo (19).

Una posible explicación a este hallazgo puede deberse a la presencia de gérmenes orales patógenos involucrados en la enfermedad dental, como Porphyromonas gingivalis y Treponema denticola, cuyo ADN ha sido encontrado en tejidos placentarios de mujeres con desórdenes hipertensivos, tal como se evidenció en el estudio realizado por Chaparro et al., OR 9,39 (IC95 \%= 2,39-36,88) para T. denticola y OR de 7,59 (IC95 \%= 1,39 - 41,51) para P. gingivalis (20); sin embargo, las anteriores bacterias se han relacionado mucho más con la gingivitis que con la caries, pero deja en claro que la microbiota oral puede tener un papel importante en el desarrollo de la preeclampsia.

Uno de los mecanismos fisiopatológicos propuestos es el de la colonización de la placenta por bacterias orales transportadas por vía hematógena causando alteraciones en el desarrollo normal placentario y generando aterosis $(21,22)$. Otro mecanismo es el de cambios inmunológicos sistémicos ocasionados por la enfermedad dental que llevan a complicaciones durante el embarazo (23).

Hay evidencia contradictoria en la literatura frente al papel del tratamiento de la enfermedad dental en la disminución del riesgo para preeclampsia. Un estudio realizado por Boggess et al., encuentra que mujeres con historial de tratamiento periodontal tiene un riesgo de 3,71 veces más de desarrollar preeclampsia severa que aquellas sin historial (24).

Otro estudio en el que se pretendió reconocer el papel del tratamiento de la enfermedad periodontal en la prevención de la preeclampsia no encuentra asociación, OR $0,82($ IC95 \% $=0,44-1,56)(\underline{25})$.

En nuestro estudio las pacientes de etnia blanca mostraran una asociación significativamente estadística con la preeclampsia, algo que parece no evidenciarse en otros estudios. Según la literatura, son las pacientes afrodescendientes quienes tienen mayor riesgo de padecerla (26). Se considera que debido al alto mestizaje de la población colombiana, es complejo garantizar un buen discernimiento del real genotipo y fenotipo de un paciente, por lo que este hallazgo podría estar influenciado por tal factor.

En este estudio hubo limitaciones en la obtención de la información de la variable etnia, con datos perdidos en esta variable de 9,4\%. Sólo una minoría de las pacientes del estudio tuvo urocultivo y evaluación por odontología en todos los trimestres del embarazo. Se debe aclarar que el 91,8 \% de las pacientes no tenían antecedente de hipertensión arterial, por lo que deberá ser evaluado por otros estudios. 
Deberán realizarse estudios para identificar la asociación de cada una de las bacterias de la flora encontrada en la infección periodontal y dental y el desarrollo de preeclampsia.
Aunque múltiples estudios continúan apuntando hacia la enfermedad periodontal y dental como uno factores con mayor asociación para el desarrollo de preeclampsia, se deberá continuar indagando en este factor, que no sólo ha mostrado asociación con preeclampsia sino con otras enfermedades y síndromes (27).

Deberán realizarse estudios para identificar la asociación de cada una de las bacterias de la flora encontrada en la infección periodontal y dental y el desarrollo de preeclampsia.

A partir de la evidencia obtenida se reconoce que en la población del Valle de Aburrá, la presencia de caries durante el primer trimestre del embarazo puede ser relevante como factor de riesgo para preeclampsia cuando ocurre concomitantemente con infecciones urinarias y otros hallazgos relacionados con infección dental. Esto lleva a reforzar la recomendación de propiciar a toda gestante la evaluación odontológica al ingreso del control prenatal.

\section{Agradecimientos}

A la gerencia de la CICCC y al equipo de Promoción y Prevención, quiénes prestaron su apoyo para la construcción de la base de datos que sirvió para el desarrollo del actual estudio.

\section{Bibliografía}

1. Duley L. The global impact of Pre-eclampsia and Eclampsia. Semin Perinatol. 2009; 33(3): 130-137. link

2. Say L, Chou D, Gemmill A, Tunçalp Ö, Moller A-B, Daniels J, et al. Global causes of maternal death: a WHO systematic analysis. Lancet Glob Health. 2014; 2(6): e323-333. link

3. Kassebaum NJ, Bertozzi-Villa A, Coggeshall MS, Shackelford KA, Steiner C, Heuton KR, et al. Global, regional, and national levels and causes of maternal mortality during 1990-2013: a systematic analysis for the Global Burden of Disease Study 2013. Lancet. 2014; 384(9947): 980-1004. link

4. Departamento Administrativo Nacional de Estadística (DANE) [Sitio de Internet]. Hallado en URL: http://www.dane.gov.co/index.php/esp/component/content/article/118-demograficas/estadisticas-vitales/4675-defunciones-no-fetales-2013

5. American College of Obstetricians and Gynecologists, Task Force on hypertension in pregnancy. Hypertension in pregnancy. Report of the American College of Obstetricians and Gynecologists' Task Force on Hypertension in Pregnancy. Obstet Gynecol. 2013; 122(5): 1122-1131. link

6. Paré E, Parry S, McElrath TF, Pucci D, Newton A, Lim K-H. Clinical risk factors for preeclampsia in the 21st century. Obstet Gynecol. 2014; 124(4): 763-770. link

7. Juarte ER, Bonora RB, Hernández AP. Factores de riesgo de la enfermedad hipertensiva del embarazo. Rev Arch Méd Camagüey. 2014; 10(5). link 
8. Cruz Hernández J, Hernández García P, Yanes Quesada M, Isla Valdés A. Factores de riesgo de preeclampsia: enfoque inmunoendocrino. Parte I. Rev Cuba Med Gen Integral. 2007; 23(4): 0-0. link

9. Ha J-E, Jun J-K, Ko H-J, Paik D-I, Bae K-H. Association between periodontitis and preeclampsia in never-smokers: a prospective study. J Clin Periodontol. 2014; 41(9): 869-874. link

10. Conde-Agudelo A, Villar J, Lindheimer M. Maternal infection and risk of preeclampsia: systematic review and metaanalysis. Am J Obstet Gynecol. 2008; 198(1): 7-22. link

11. Huang X, Wang J, Liu J, Hua L, Zhang D, Hu T, et al. Maternal periodontal disease and risk of preeclampsia: a meta-analysis. J Huazhong Univ Sci Technol Med Sci. 2014; 34(5): 729-735. link

12. Matuszkiewicz-Rowińska J, Małyszko J, Wieliczko M. Urinary tract infections in pregnancy: old and new unresolved diagnostic and therapeutic problems. Arch Med Sci AMS. 2015; 11(1): 67-77. link

13. Kalakonda B, Koppolu P, Baroudi K, Mishra A. Periodontal systemic connections-novel associations-a review of the evidence with implications for medical practitioners. Int J Health Sci. 2016; 10(2): 293-307. link

14. Martín-Vegue AR, Vázquez-Barquero J, Castanedo SH. CIE-10 (I): Introducción, historia y estructura general. Papeles Méd. 2002; 11(1): 24-35. link

15. Asociación Médica Mundial. Declaración de Helsinki. Principios éticos para las investigaciones médicas en seres humanos. Seul Corea AMM. 2008. link

16. Consejo de Organizaciones Internacionales. Pautas éticas internacionales para la investigación biomédica en seres humanos [Sitio en Internet]. 2002. Hallado en URL: http://www.ub.edu/rceue/archivos/Pautas Eticas Internac.pdf

17. Minassian C, Thomas SL, Williams DJ, Campbell O, Smeeth L. Acute maternal infection and risk of preeclampsia: a population-based case-control study. PloS One. 2013; 8(9): e73047. link

18. Shamsi U, Hatcher J, Shamsi A, Zuberi N, Qadri Z, Saleem S. A multicentre matched case control study of risk factors for preeclampsia in healthy women in Pakistan. BMC Womens Health. 2010; 10(1), 1. link

19. Khader Y, Jibreal M, Burgan S, Amarin Z. Risk indicators of pre-eclampsia in north Jordan: is dental caries involved? Gynecol Obstet Invest. 2007; 63(4): 181-187. link

20. Chaparro A, Blanlot C, Ramírez V, Sanz A, Quintero A, Inostroza C, et al. Porphyromonas gingivalis, Treponema denticola and toll-like receptor 2 are associated with hypertensive disorders in placental tissue: a case-control study. J Periodontal Res. 2013; 48(6): 802-809. link 
21. Barak S, Oettinger-Barak O, Machtei EE, Sprecher H, Ohel G. Evidence of periopathogenic microorganisms in placentas of women with preeclampsia. J Periodontol. 2007; 78(4): 670-676. link

22. Swati P, Thomas B, Vahab SA, Kapaettu S, Kushtagi P. Simultaneous detection of periodontal pathogens in subgingival plaque and placenta of women with hypertension in pregnancy. Arch Gynecol Obstet. 2011; 285(3): 613-619. link

23. Han YW. Oral health and adverse pregnancy outcomes - What's next? J Dent Res. 2011; 90(3): 289-293. link

24. Boggess KA, Berggren EK, Koskenoja V, Urlaub D, Lorenz C. Severe preeclampsia and maternal self-report of oral health, hygiene, and dental care. J Periodontol. 2013; 84(2): 143-151. link

25. Newnham JP, Newnham IA, Ball CM, Wright M, Pennell CE, Swain J, et al. Treatment of periodontal disease during pregnancy: a randomized controlled trial. Obstet Gynecol. 2009; 114(6): 1239-1248. link

26. Paré E, Parry S, McElrath TF, Pucci D, Newton A, Lim K-H. Clinical risk factors for preeclampsia in the 21st century. Obstet Gynecol. 2014; 124(4): 763-770. link

27. Gulati M, Anand V, Jain N, Anand B, Bahuguna R, Govila V, et al. Essentials of Periodontal Medicine in Preventive Medicine. Int J Prev Med. 2013; 4(9): 988-994. link 